\title{
REFERENCE SERIES
}

\section{Air Force Combat Wings}

Lineage and Honors Histories

1947-1977

Charles A. Ravenstein

United States Air Force Historical Research Center

OFFICE OF AIR FORCE HISTORY

UNITED STATES AIR FORCE

WASHINGTON, D.C., 1984 